\title{
Asynchronous Web Technology in Online Counselling System
}

\author{
Siti Nurulain Mohd Rum*, Abu Bakar Md Sultan, Razali Yaakob and T. Perumal \\ Faculty of Computer Science and Information Technology, University Putra Malaysia; \\ snurulain@upm.edu.my
}

\begin{abstract}
Objectives: To identify the users' requirements of the Online Counselling System, to develop an AJAX-based Online Counselling System based on the analysed requirements and to measure its effectiveness. Methods / Statistical Analysis: This research work comprises a series of studies. Firstly, the questionnaire survey was conducted to understand the users' requirements. The target respondents were students at a university in Malaysia and 170 participated in this study. Secondly, the development of an AJAX-based Online Counselling System based on the analysis users' requirements and lastly, the evaluation of the developed Online Counselling System through the usability testing, conducted to see its effectiveness. Findings: Finding a research work that specifically measuring an AJAX-based application in terms of users' satisfaction is lacking and could not be located. Therefore, this research work was conducted to measure an AJAX-based application system. In this study, we have used the usability testing as the user-centered technique to measure the effectiveness of the AJAX-based Online Counselling System. The Online Counselling System is an interactive and responsive application act as an alternative platform to the face-to-face counselling approach. There are four components covered in usability testing, namely, learnability, efficiency, memorability and satisfaction. The result shows that the Online Counselling System has met the user requirements, measured based on these four criteria. This could only be achieved through the use of AJAX approach development that provides a minimal page reloading and very suitable for an interactive web-based application system. This research work finding provides the evidence that the AJAX architecture principle offers a great promise for developers to develop a more interactive and responsive application without compromising on performance of the application
\end{abstract}

Keywords: Asynchronous Web Technology, Counselling, Online Counselling System, AJAX, XMLBROWSER

\section{Introduction}

It is an undeniable fact that advances in technology are forever changing the way people work. The computer in general and the internet in particular, has not only provided access to huge amounts of information, but also revolutionized the way people communicate with one another. Email, instant messaging and other technologies offer people the opportunity to exchange ideas and information and across geographical areas. The proliferation of computer mediated communications has resulted the field of psychotherapy to experiment the use of these technologies for what has come to known as online counselling. Similar to the other type of therapy such as occupational therapy and physical therapy, online counselling helps an individual to tackle issues concern to them in life under the guidance of a professional. Online therapy does not diagnose medical disorders, but it is similar to the idea of coaching and helping a person to address specific concerns with specific skills. There are two main advantages of using online counselling, firstly it can potentially increase accessibility, especially to those who suffer from extreme shyness, social anxiety or other such disorders, secondly, anonymity and invisibility give privacy to those who have stigma. To deliver the mental health services via online, a highly interactive system is needed. A highly interactive system has specific characteristics firstly, the application should be able to visualized data in different windows using different techniques tex-

${ }^{*}$ Author for correspondence 
tually or graphically, secondly, the states changes of data should immediately reflect program, thirdly, changes of user interface should be easily done and lastly, modifying the look-and-feel of the system should be possible without major effect. To realize these requirements, AJAX offers such great promises for interactive and large scale development of web based application. The minimal page reloading introduced by AJAX makes it very appealing for many web developers. The architectural properties in AJAX provide the functions in order to deal with system qualities ${ }^{1}, \underline{2}$. The intrinsic of architectural properties provided by $\mathrm{AJAX}^{\underline{3}}$ are as follows:

\subsection{User Interactivity}

In the literature on Human Computer Interaction, defined interactivity as the degree in which subjects (i.e. human being or possibly another living creature or a computer program) in a communication process has the ability to control the flow of information and can exchange roles in their mutual discourse. User's perceived satisfaction towards a web site result from the interactivity level, the higher the better ${ }^{6}$. Improving these properties has been the key motivating factor of AJAX movement ${ }^{3}$.

\subsection{User-perceived level}

User-perceived latency can be defined as the delay between the user input and computer response ${ }^{3}$. Generally, to improve user-perceived performance, there are two primary methods ${ }^{\underline{3}}$. First, reduce the round-trip time by defining the length of time for a message from the user to send a request and response of the server over the network. Secondly, enable the asynchronous interaction between users and application ${ }^{3}$.

\subsection{Network Performance}

Network performance refers to the measures of network service quality and it's usually measured by throughput, latency, and jitter. Throughput is the rate of data dispatched over the network and the maximum rate of data can be processed across network. The latency refers to the delay that happens in data in communication to be decoded and transmitted across network ${ }^{3}$. Reducing granularity and the amount of transmitting data can improve network performance ${ }^{3}$.

\subsection{Simplicity}

Simplicity in application refers to the quality of being simple and effort needed by a user to understand the design, implement, expand and maintain an application. It is an important principle of design in any web-bases application. Simplicity is about going deep into a user's understanding and mind to design a system that rids the inconsequential elements the means to achieve user's goals through the system ${ }^{3}$.

\subsection{Scalability}

Scalability in distributed environments refers to the ability of a system to deal with a growing number of system's components $s^{z}$. In web engineering field, a system's scalability is determined by the degree through which, the ability of the system to continue to function well without affecting the performance when it changed its scope or size to serve clients ${ }^{\underline{7}}$. Web architecture with scalable design can easily be configured to serve an increased number of client requests ${ }^{\underline{p}}$.

\subsection{Visibility}

Visibility ${ }^{\underline{8}}$ refers to the degree to which the application enables ease of operation without instrumental assistance. In AJAX frameworks, the interaction of client-server is low in terms of visibility, as they are based on proprietary protocols ${ }^{-}$. The better visibility of an application makes the client-server interactions more effective. However, too much of visibility can also contribute to negative effects, especially on security concern. Thus, low visibility in a system is not less worthy characteristic per se, depending on the system requirement property and trade-offs made ${ }^{8}$.

Online counselling refers to the provision of psychological interventions conducted over the internet, either synchronously or asynchronously. It can also be conducted either as an individual or group setting? Asynchronous forms of communication can be identified by the lag in time between contacts, whereas synchronous communication occurs in real-time $e^{10}$. As such, methods of communication which do not regularly provide instantaneous responses, such as emails and forums classified as asynchronous. The communication modalities that allow for real-time communication, such as chat (e.g., MSN) or video (e.g., Skype) can be classified as synchronous type of communication. 11 define a web-based intervention as a primarily self-guided mental health related 
intervention that is operated by a prescriptive online program. The program is designed to improve knowledge as well as to create positive change of understanding and awareness by the provision of quality health-related information through the use of interactive web-based components. Thus, web-based programs can include education interventions (e.g., programs which provide information regarding the associated features of a mental disorder, explanation of symptoms, possible causes, its effects, and treatment options), self-help therapeutic interventions (e.g., Treatment or prevention, self-guided online programs designed to promote positive cognitive, behavioural, and emotional change), and therapist supported interventions, (e.g., similar to self-help interventions, but with a mental health professional to provide support and guidance). There is increasing evidence to suggest that some psychological services provided online are as effective, or almost as effective as, similar services provided by face-to-face ${ }^{12}$. In the past, most of the telehealth communication was done via desktop applications in a decentralized approach using a peer-to-peer system. An example to this are LivePerson ${ }^{13}$, Kids Helpline ${ }^{14}$ and eheadspace $\frac{15}{5}$ and Web peer $\frac{16}{}$. Today there are number of audio and video technology related to web-chat solutions, and most of them are based on Java applets ${ }^{17}$. Applets are known for their common security problem across browsers ${ }^{18}, \underline{19}$. AJAX came to change the landscape. This technology has dramatically expanded the capacity for person to person communication and has attracted attention. Online counselling appears to be a growth industry. The synchronous or simultaneous form of online counselling is known as instant messaging, which can manifest itself in four ways ${ }^{20}$. Strictly text based is the first form, while the second is text which includes an avatar. An avatar is a movable graphic that represents a person in a virtual reality environment ${ }^{20}$. The purpose of an avatar is to personalize text-based communication and make the exchange friendlier, and in some cases more realistic ${ }^{20}$. The third form of instant messaging requires the parties or a group of people involved to enter into a virtual reality chat room and can control the atmospheric conditions as well as the characters that represent them ${ }^{20}$. The final form of instant messaging involves incorporating a webcam so that two or more people can see each other on their computer screens as they communicate by typing ${ }^{20}$. Figure $1^{21}$ shows the pictorial representation of the types of telehealth or online counselling system. Synchronous web chat applications can be classified as very interactive, as they provide a facility through which users can exchange messages, often very frequently.

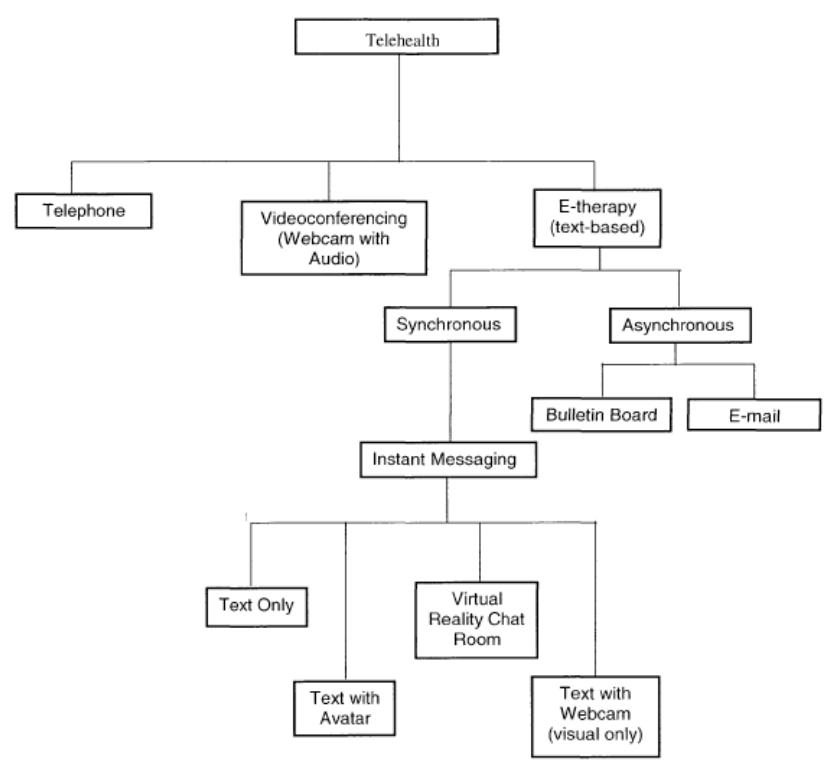

Figure 1. The pictorial representation of the types of telehealth system.

For some instant messaging applications, to compose, store, update and display message exchange between users requires frequent page reloading. This may cause the degradation of system performance and potentially to present security issues. Today using AJAX is an obvious approach to avoid unwanted page reloads. This can be done by AJAX, which allows small amounts of data to be exchanged in web pages. The nature of web-based counselling system can be classified as a very responsive and interactive type of application that really suitable with the characteristics of AJAX. By applying AJAX, the prototype system follows the SPIAR architectural model ${ }^{21}$ as shown in Figure $2 \underline{22}$. According to ${ }^{8} \underline{\underline{23}}$ and $^{24}$, the main elements of SPIAR-based architectural divided into three categories, namely processing elements, data elements, and connecting elements. An overview of these elements is shown in Figure 2. The processing elements refer to the components (e.g. Client browser, AJAX engine, server application and etc.) that provide transformation of the data elements. The data elements consist of information to be used for processing elements during the transformation process. The connecting elements functioned as the glue that keeps the components together by enabling them to be interacted each other. 


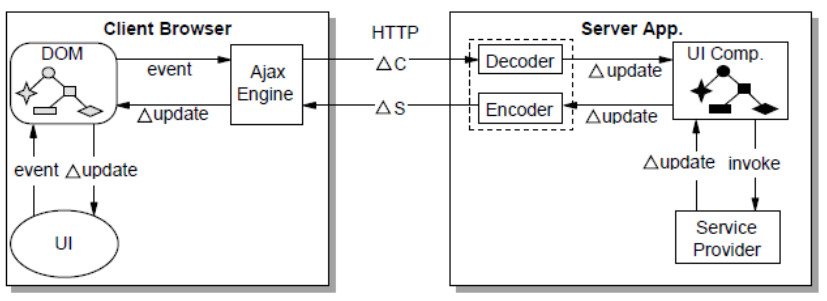

Figure 2. Processing View of SPIAR-based architecture.

With the advent of technology in Web 2.0 and AJAX, to make a web-based application behaves similar to traditional desktop applications is possible. Many popular applications used AJAX including Flickr, Waze, Google Maps and Google Docs. The Webs 2.0 advanced in terms of its ability to access and modify the DOM inside the XHTML document. This can be done without reloading entire web page. In terms of graphical user interface, the dynamic elements of Document Object Model (DOM) allow a web application behave similar to a desktop application. However, there are several issue poses by Web 2.0 when it comes to security vulnerabilities. AJAX was introduced to address these issues. AJAX isn't a technology, but it is a combination of several technologies and each flourishing in its own right, coming together in powerful new ways. These technologies are CSS, HTML, DOM, XML, XMLHttpRequest, XSLT and JavaScript combined together as AJAX package that makes web-based application more interactivity and easy navigation..$^{22}$ The purpose of to have standard Hypertext Markup Language (HTML) and Cascading Style Sheets (CSS) in AJAX are to style and mark-up information in dynamic web pages. The combination of JavaScript and DOM in AJAX can be used to display web content dynamically. AJAX is a set of technologies innovated to improve the functionality and the appearance of traditional web sites $\underline{25}$. It depends on the nature of XMLBrowserRequest $\frac{26}{}$, DOM, CSS and other technologies ${ }^{25}$. The common features of AJAX had been used in libraries like ActiveX, Java applet and Flash ${ }^{25}$. To asynchronously retrieve and request data from remote servers, only necessary data will be sent over and AJAX used JavaScript to do these tasks ${ }^{26}$. AJAX used XML to collect text-style or numerical data from the browser. It also used JavaScript to extract data from XML and HTML. The pattern of a classical style of a typical web application versus web application using AJAX is presented in Figure 3.

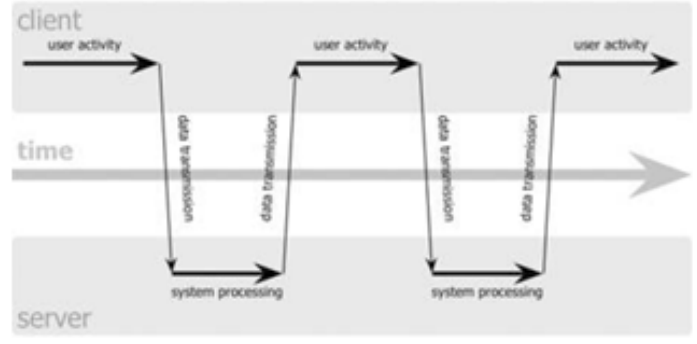

\section{Ajax Request}

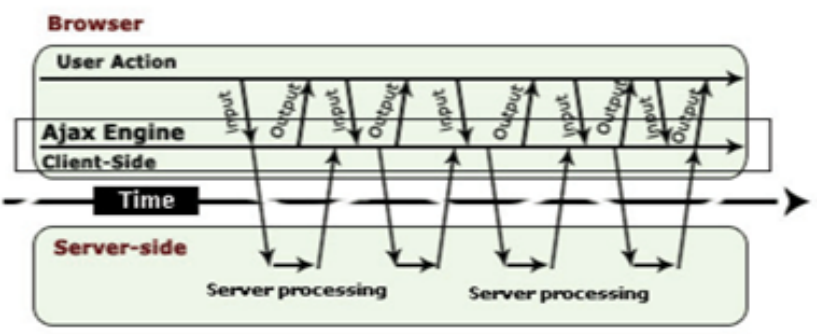

Figure 3. The classical web application (top) vs AJAX(bottom).

Although there are a number of web-based applications applied AJAX, research work that particularly focused on the implementation of AJAX in web-based application development and measuring its effectiveness is lacking. In this research work a series of studies were conducted with the objective, firstly, to identify the acceptance level of online counselling system, secondly to design and develop a prototype system of online counselling system using AJAX approach and lastly to measure the effectiveness of the prototype system using the usability test.

\section{Research Method}

This research work comprises a series of studies; firstly, the exploratory questionnaires related to the system functional requirements were designed to get a better understanding of the users' acceptance level, users' need, secondly the development of prototype system and lastly the evaluation of the prototype system. The questionnaire survey is divided into four sections, the first section is regarded as the profile section of respondents, and the rest are regarded as the software architectural properties namely user-perceived level, userinteractivity, simplicity and scalability. In user-perceived level and user-interactivity sections, respondents are asked about their preferred mode of internet counselling session and two options are given for them to choose; a private session or a group session. In a private session, a counsellor is allowed to attend only one student at a time in isolated session. 
In a group session, a counsellor is allowed to attend a group of student at a time in non-isolated session. In simplicity section, respondents are asked about their preference of style to express emotions via internet counselling. There are options provided for the respondents to answer that question such as using accentuations of voice, for example, using ${ }^{\star}$ ANGER $^{\star}$ to express a vocal emphasis, acronyms (e.g., lol for "laugh out loud") and emoticons, trailers such as using "as...uh...," and "um." to represent a hesitation or break in one's train of thought, parenthetical expressions such as using sigh or "not sure how I feel" to indicate body language or thoughts and feelings). In scalability section, respondents were asked about their opinion about system's scalability feature. The target population has been identified as students from a university in Malaysia. A convenience sampling techniques were conducted to gather the information. In this non-probability sampling technique, the respondents were selected based on convenient accessibility and proximity to the researchers. In this study the invitations were broadcasted through email, the target sample for this study is 170 students. The pilot test was conducted with the objectives; firstly, to see the effectiveness of the designed questions to avoid a misunderstanding of the questionnaires, secondly, to ensure the designed questions are not ambiguous and lastly to check the instructions given are clear as well as to test how long it takes for respondents to complete. The distribution of respondents is presented in Table 1.

Table 1. Respondents distribution by faculties

\begin{tabular}{|l|c|c|}
\hline Faculties & $\begin{array}{c}\text { Total } \\
\text { Respondents }\end{array}$ & $(\%)$ \\
\hline Faculty of Arts and Social Sciences & 12 & $8 \%$ \\
\hline Faculty of Business and Accountancy & 5 & $3 \%$ \\
\hline $\begin{array}{l}\text { Faculty of Computer Science \& } \\
\text { Information Technology }\end{array}$ & 37 & $24 \%$ \\
\hline Faculty of Dentistry & 3 & $2 \%$ \\
\hline $\begin{array}{l}\text { Faculty of Economics \& } \\
\text { Administration }\end{array}$ & 16 & $10 \%$ \\
\hline Faculty of Education & 17 & $11 \%$ \\
\hline Faculty of Engineering & 12 & $8 \%$ \\
\hline Faculty of Languages and Linguistics & 10 & $6 \%$ \\
\hline Faculty of Law & 9 & $6 \%$ \\
\hline Faculty of Medicine & 11 & $7 \%$ \\
\hline Faculty of Science & 16 & $10 \%$ \\
\hline Faculty of the Built Environment & 7 & $4 \%$ \\
\hline Other & 2 & $1 \%$ \\
\hline Total & 157 & $100 \%$ \\
\hline
\end{tabular}

The data gathered from the survey are analysed and transformed into system features. These features were designed and implemented in the prototype system to fulfil the users' need. Table 2 provides the summary of the functional requirements mapped to related AJAX functions. The oracle database is used to store users' information and the messages exchanged between users. The server side scripting language named Hypertext Preprosessor (PHP) was used to code the prototype system. This programming language is used to code the application because it has fast load time, it is database independence and compatible with many commonly used database and it is easy to learn the language. In the prototype system, the function to compose and display messages exchanged between users are handled by AJAX in the backend process. This process requires minimal page reloading to avoid interference of the current state of the web page. It used XMLBrowserRequest to send and receive data from the server to the client. Figure 4 presents a part of AJAX script used in the prototype system.

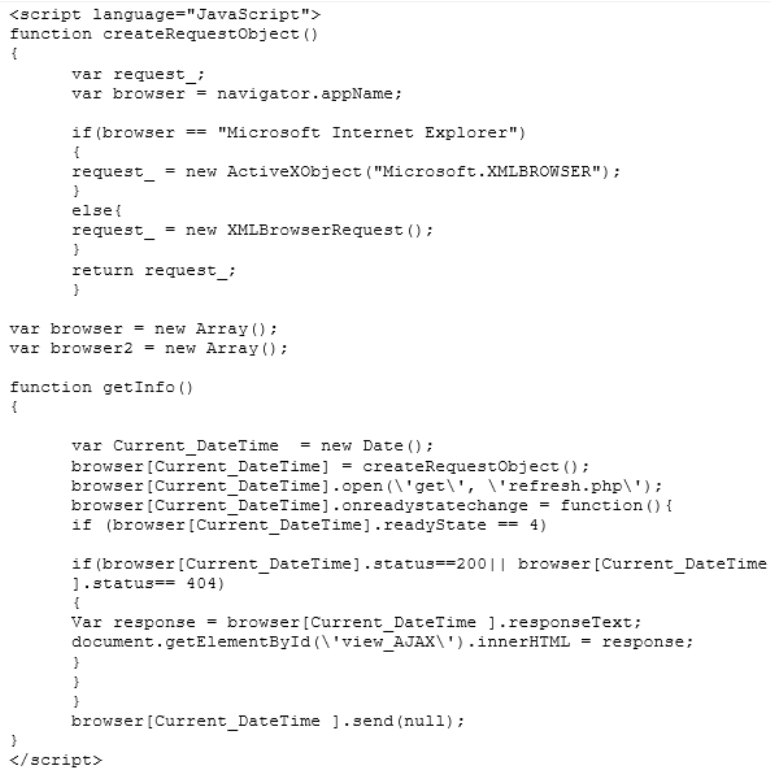

Figure 4. Part of AJAX script using JavaScript.

XMLBrowserRequest was first built by Microsoft and today it has been employed by many modern browsers such as Google, Apple and Mozilla. It has been standardized in World Wide Web consortium (W3. The 'browser' variable is an array object was created to store data about browsers platform. The function named CreateRequestObject is 
responsible to create the XMLHttpRequest to process the AJAX requests. The readyState function holds the status of XMLHttpRequest. The value varies from 0 to 4 where value 1 gives the indication that the server connection is established, value 2 shows that the request is received and value of 3 gives the indication that the XMLHttpRequest is processing the request and value 4 shows the response is ready. The innerHTML properties will change or returns the HTML contents in the web browsers if the request completed and the response is ready to be received by the server. There will be no processes executed if the value of readyState is 1, 2 or 3 . The architecture of prototype system is presented in Figure 5.

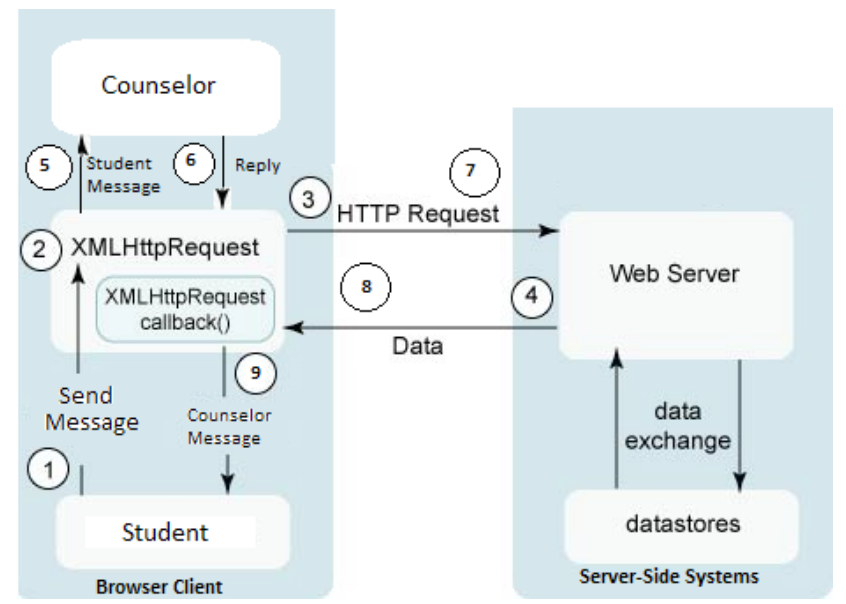

Figure 5 Online Counselling Architecture using AJAX approach.

The architecture of the prototype system is presented in Figure 5. The processes presented as no 1 and 6 refer to the process of exchanging messages between student and counsellor. When the submit button clicked by users, an event handler will be evoked. A JavaScript can be executed when an event occurs. The handler's code creates an XMLHttpRequest object to interact with server and this process is presented as no 2. This allows the update and refresh process be executed on part of the web page without disrupting what the user is doing. During this process only a small amount of data are exchanged and displayed at users' screen. In processes 3 and 7, the XMLHttpRequest object will requests data from server. The server will retrieve the related data and send it over to the users. In Figure 5, these processes indicated as processes no
4 and 8. The XMLHttpRequest fires an event immediately when the data arrived and this process often called a callback process (processes 6 and 9).

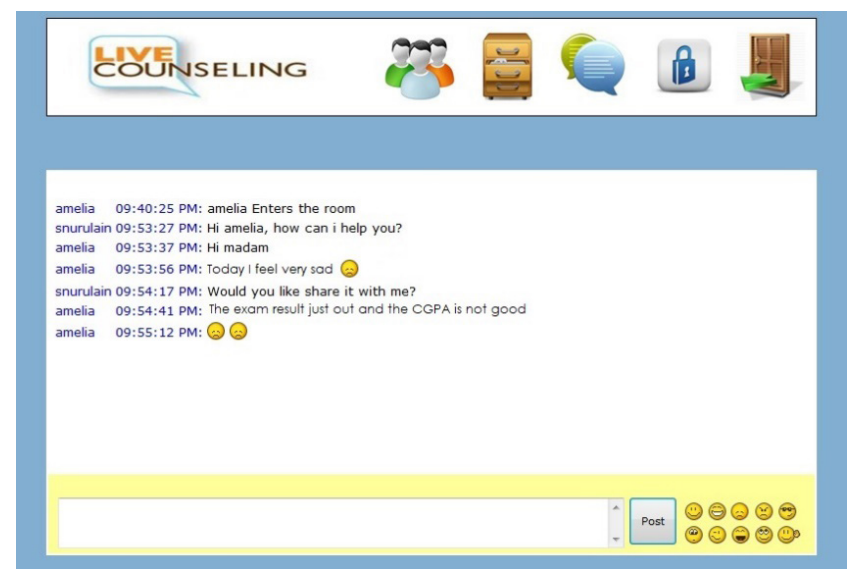

Figure 6 The final look of online counselling web page.

The final look of the prototype system is presented in Figure 6. This system allows users to compose text messages to be exchanged between users. With the growing of social media technology, emoticons have become a new form of language used to represent emotions. Studies revealed that emoticons have the possibility to enhance online communication ${ }^{27-29}$. In this study, the emoticons are provided as an additional feature in the prototype system to help users to express their emotions while communicating. The effect of using emoticons in computer mediated communication has been performed by many $y^{30-32}$. It is proven that emoticon has the ability to represent the actual expression of a person similar to face to face communication with respect to social context. There are ten different types of emoticons provided in the prototype system. These emoticons are commonly used in computer mediated communication to express a different mood and feeling of a person such as happiness, sadness, angriness and confusion. The 'Post' button allows the messages exchanged between users to be displayed in the dashboard area. The nickname of the sender as well as the timestamp of each communication will also displayed. With the timestamp, users can see when the one last said something. 
Table 2. The function requirements of Online Counselling

\begin{tabular}{|l|l|l|l|}
\hline $\begin{array}{l}\text { Architectural } \\
\text { Properties } \\
\text { (Theme) }\end{array}$ & Result of the Analysis & System features & AJAX function \\
\hline $\begin{array}{l}\text { User-perceived } \\
\text { level } \\
\text { \& } \\
\text { User Interactivity }\end{array}$ & $\begin{array}{l}\text { 80\% of respondents' preferred the } \\
\text { private session to interact with } \\
\text { counsellor }\end{array}$ & $\begin{array}{l}\text { The private virtual chat room } \\
\text { in real-time session where } \\
\text { user will be treated by a } \\
\text { counsellor. }\end{array}$ & $\begin{array}{l}\text { Allow message exchange between users } \\
\text { instantly. Each session is protected } \\
\text { with unique session ID for privacy } \\
\text { purpose. }\end{array}$ \\
\hline Simplicity & $\begin{array}{l}\text { 75\% of respondents preferred } \\
\text { to have emoticons to express } \\
\text { emotion while communicating }\end{array}$ & $\begin{array}{l}\text { The pictorial representation of } \\
\text { a facial expression are used to } \\
\text { express a person's mood stored } \\
\text { in database }\end{array}$ & $\begin{array}{l}\text { Allow text strings to be replaced with } \\
\text { appropriate emoticons to represent } \\
\text { emotions of a user }\end{array}$ \\
\hline Scalability & $\begin{array}{l}\text { 95\% of respondents agreed that } \\
\text { there should be no limits on } \\
\text { the number of users' using the } \\
\text { system concurrently }\end{array}$ & $\begin{array}{l}\text { Support multi users and } \\
\text { concurrent access sessions }\end{array}$ & $\begin{array}{l}\text { AJAX encourages the adoption of } \\
\text { XMLTTPR request to facilitates the } \\
\text { hitting process on servers without the } \\
\text { need of user interactions. }\end{array}$ \\
\hline
\end{tabular}

\section{Result}

User testing is done at real environment with the targeted platforms (browser, the hardware and the software). In this research work, the usability testing is used to measure the effectiveness of the prototype system. Usability testing is a black-box technique used in user-centered interaction design. This test has been used by many such as $\frac{33-36}{t o}$ measure the efficiency, effectiveness and satisfaction of a software. The usability testing covers four components, namely learnability (e.g. how easy to perform a task in the system for the first time use?), efficiency (e.g. how fast one's can experience in performing a basic task in the system?), memorability (e.g. how easy to remember the design of the system for a certain period of time not using it?) and satisfaction (e.g. how much user like to use the system?). The advantages of using usability test, firstly, it facilitates the software developer to discover potholes in prototype system as well as to discover the bugs that usually visible, secondly, it is very economical yet highly effective and thirdly, the potholes and the bugs can be fixed before the system is finally release to the end user
User testing was conducted at the computer lab. The participants are divided into two groups, counsellor and student. There are five counsellors and five students participated in this study. A unique username and password is created for each of them in order to access the prototype system. The counsellor module was tested by group of counsellor whereas student module was tested by a group of student. During the testing session they are require to communicate in pair through the prototype system where each pair consist student and counsellor (one-to-one session). There are five parallel sessions conducted concurrently. Each session consumed about 30 minutes to complete. For the first ten minutes, respondents are allowed to explore the system to get them familiar. Towards the end of the session, all participants are required to fill up the system evaluation form. The result of a usability test is presented in Table 3. From the results obtained, in overall, it can be concluded that most of the participants satisfied with the prototype system in measuring the user friendliness of the system, the look and feel of the system and the response time for exchanging messages between users as well as the usefulness of the system.

Table 3. Usability test result

\begin{tabular}{|l|l|}
\hline Application's Attributes & Feedback \\
\hline User Friendly & 85\% of respondents agreed that system is easy to use. Only 15\% indicate it's as an average. \\
\hline $\begin{array}{l}\text { User Interactivity and Layout } \\
\text { Design }\end{array}$ & All respondents agreed that the User Interface and Layout design is simple and easy to use. \\
\hline Text Style & All respondents satisfied with the text style used by the system \\
\hline Navigation & All respondents satisfied with the structure of the system \\
\hline Response time & All respondents satisfied with the response time \\
\hline System Usefulness & $\begin{array}{l}\text { All respondents agreed that the system is very useful and can be treated as an alternative to face } \\
\text { to face counselling }\end{array}$ \\
\hline
\end{tabular}




\section{Conclusion}

It has been noted in the literature that computer mediated communication using instant messaging is an effective alternative to face-to-face counselling. The literature also revealed that using online intervention in counselling can potentially bridge the gap between people and mental health service. Technology is the key enabler for online counselling and AJAX is the one of it. AJAX isn't a technology, but it is a combination of several technologies and each flourishing in its own right, coming together in powerful new ways. These technologies are CSS, HTML, DOM, XML, XMLHttpRequest, XSLT and JavaScript combined together as an AJAX package to make web based application more interactivity and easy navigation. The minimal page reloading offered by AJAX makes it very appealing to be used by many web based application development. In this study, we have used AJAX for building a web-based counselling system. This highly interactive system provides a platform for the mental health services to be conducted via online. There are two main modules provided by the system one is used by counsellors and another is for students. The system allows a counselor and a student to exchange, communication and information instantly. By using AJAX, many functions provided in the prototype system can be handled in single web page that is impossible to be done in traditional web-based application development. To measures the effectiveness of the prototype system, the usability test was conducted. Six questions were designed to measure users' satisfaction pertaining to the user-friendliness of the application, user-interactivity, the look and feel of the application, user-navigation, response time and the usefulness of the application. The result shows that most of users satisfied with the prototype system. Although JavaScript is labelled as a secure HTML programming script and has been used extensively by websites for a long period of time, some web surfers preferred to turn off JavaScript for its potential to present compatibility issues and leave a system or a network exposed to security vulnerabilities. The disabled property of JavaScript in the web browser has always caused the dysfunctional of AJAX features. This is one of the problems encountered during the usability test. In this study, we also found other related issues of using AJAX; it requires a complex code and it is difficult to debug, maintain and to test particularly in the situation where the internet connection is slow or unreliable. A workaround to this drawback is suggested for future enhancement of the application is to use a parallel code by combining JavaScript and nonJavaScript codes to produce dynamic web pages to address the problem of dysfunctionality of AJAX.

\section{Reference}

1. Bass L, Clements P,Kazman R. Software architecture in practice: Addison-Wesley Longman Publishing Company, 2003.

2. Offutt J. Quality attributes of web software applications. IEEE Software special issue. 2002 March/April;19(2):2532. Crossref.

3. Mesbah A. Analysis and Testing of Ajax-based Singlepage Web Applications [Unpublished Ph.D thesis]. Delft University of Technology; 2009.

4. Lazar J, Feng JH, Hochheiser H. Research methods in human-computer interaction: Morgan Kaufmann; 2017.

5. Norman KL. Cyberpsychology: An introduction to humancomputer interaction: Cambridge university press; 2017.

6. Teo HH, Oh LB, Liu C, Wei KK. An empirical study of the effects of interactivity on web user attitude. International Journal of Human-Computer Studies. 2003; 58(3):281-305. Crossref.

7. Netcraft. January 2009 Web Server Survey2011. January 2009; Available from: Crossref.

8. Fielding RT. Architectural styles and the design of networkbased software architectures: University of California, Irvine; 2000.

9. Barak A, Klein B, Proudfoot JG. Defining internet-supported therapeutic interventions. Annals of Behavioral Medicine. 2009; 38(1):4-17. Crossref. PMid:19787305

10. Perle JG, Langsam LC, Nierenberg B. Controversy clarified: An updated review of clinical psychology and tele-health. Clinical psychology review. 2011; 31(8):1247-58. Crossref. PMid:21963670

11. Barak A, Grohol JM. Current and future trends in internet-supported mental health interventions. Journal of Technology in Human Services. 2011; 29(3):155-96. Crossref.

12. Barak A, Hen L, Boniel-Nissim M, Shapira Na. A comprehensive review and a meta-analysis of the effectiveness of internet-based psychotherapeutic interventions. Journal of Technology in Human Services. 2008; 26(2-4):109-60. Crossref.

13. Finn J, Bruce S. The LivePerson model for delivery of etherapy services: A case study. Journal of Technology in Human Services. 2008; 26(2-4):282-309. Crossref.

14. King R, Bambling M, Reid W, Thomas I. Telephone and online counselling for young people: A naturalistic comparison of session outcome, session impact and therapeutic alliance. Counselling and Psychotherapy Research. 2006; 6(3):175-81. Crossref. 
15. Rickwood D, Webb M, Kennedy V, Telford N. Who Are the Young People Choosing Web-based Mental Health Support? Findings From the Implementation of Australia's National Web-based Youth Mental Health Service, eheadspace. Journal of Medical Internet Research. 2016; 3(3).

16. Wang Z, Chen $\mathrm{H}$, Chi Y, Xin R. Web peer counseling system. IEEE International Conference on Educational and Information Technology (ICEIT). 2010. Crossref.

17. Evans E, Rogers D. Using Java applets and CORBA for multi-user distributed applications. Journal of IEEE Internet Computing. 1997;1(3):43-55. Crossref.

18. Ganesh N. Static Analysis of Malicious Java Applets. 2015.

19. Gassen J, Chapman JP, editors. HoneyAgent: Detecting malicious Java applets by using dynamic analysis. The Americas (MALWARE). IEEE 9th International Conference on Malicious and Unwanted Software,Fajardo, PR, USA. 2014.

20. Kilroe C. A Needs Assessment of Potential Online Counselling Service Clients [Unpublished master's thesis]. University of Calgary; 2010.

21. Kilroe C. A Needs Assessment of Potential Online Counselling Service Clients: University of Calgary, Division of Applied Psychology; 2010. PMid:20668950 PMCid:PMC2977059

22. Mesbah A, van Deursen A, Lenselink S. Crawling Ajaxbased web applications through dynamic analysis of user interface state changes. ACM Transactions on the Web (TWEB). 20120; 6(1):3. Crossref.

23. Barnes JM, Garlan D, Schmerl B. Evolution styles: Foundations and models for software architecture evolution. Software and Systems Modeling. 2014;13(2):649-78. Crossref.

24. Perry DE, Wolf AL. Foundations for the study of software architecture. ACM SIGSOFT Software Engineering Notes. 1992; 17(4):40-52. Crossref.

25. Di Paola S, Fedon G. Subverting ajax. 23rd CCC Conference. 2006.

26. Garrett JJ. Ajax: A new approach to web applications. Adaptive path. February 2005.

27. Liu K-L, Li W-J, Guo M. Emoticon Smoothed Language Models for Twitter Sentiment Analysis. AAAI; 2012.

28. Liao S, Chou CY, Lin M-c. Emoticon Usage in Social Media: Influences of Social Presence and Motivation Orientation. AP-Asia-Pacific Advances in Consumer Research. 2015; 11:327.

29. Fullwood C, Orchard LJ, Floyd SA. Emoticon convergence in Internet chat rooms. Social Semiotics. 2013; 23(5):64862. Crossref.

30. Walther JB, D'Addario KP. The impacts of emoticons on message interpretation in computer-mediated communica- tion. Social science computer review. 2001;19(3):324-47. Crossref.

31. Jibril TA, Abdullah MH. Relevance of emoticons in computer-mediated communication contexts: An overview. Asian Social Science. 2013; 9(4):201. Crossref.

32. Dresner E, Herring SC. Emoticons and illocutionary force. Perspectives on Theory of Controversies and the Ethics of Communication, Springer. 2014. p. 81-90. Crossref.

33. Rum SNM, Ismail MA. Metocognitive Support Accelerates Computer Assisted Learning for Novice Programmers. Journal of Educational Technology and Society. 2017; 20(3):170-81.

34. Battleson B, Booth A, Weintrop J. Usability testing of an academic library web site: a case study. The Journal of Academic Librarianship. 2001; 27(3):188-98. Crossref.

35. Kushniruk AW, Patel VL, Cimino JJ, editors. Usability testing in medical informatics: Cognitive approaches to evaluation of information systems and user interfaces. Proceedings of the American Medical Informatics Association annual fall symposium. 1997; 218-22. PMid:9357620 PMCid:PMC2233486

36. Ben-Zeev D, Kaiser SM, Brenner CJ, Begale M, Duffecy J, Mohr DC. Development and usability testing of FOCUS: A smartphone system for self-management of schizophrenia. Psychiatric rehabilitation journal. 2013; 36(4):289. Crossref. PMid:24015913 PMCid:PMC4357360 